\title{
Variance of wind estimates using spaced antenna techniques with the MU radar
}

\author{
G. Hassenpflug, M. Yamamoto, and S. Fukao \\ Radio Science Center for Space and Atmosphere, Kyoto Unviersity, Uji 611-0011, Japan \\ Received: 3 December 2003 - Revised: 19 June 2004 - Accepted: 24 June 2004 - Published: 29 November 2004 \\ Part of Special Issue "10th International Workshop on Technical and Scientific Aspects of MST Radar (MST10)"
}

\begin{abstract}
Variance of horizontal wind estimates in conditions of anisotropic scattering are obtained for the Spaced Antenna (SA) Full Correlation Analysis (FCA) method of Holloway et al. (1997b) and Doviak et al. (1996), but are equally applicable to the Briggs method of FCA. Variance and covariance of cross-correlation magnitudes are theoretically estimated, and the standard theory of error propagation is used to estimate the variance of the wind components for the infinite SNR case. The effect of baseline orientation is investigated, and experimental data from the MU radar in Japan is presented.
\end{abstract}

Key words. Radio science (Interferometry; Remote Sensing; Instruments and Techniques)

\section{Introduction}

In the VHF band the most common methods of radar wind profiling are by Doppler Beam Swinging (DBS) and the SA technique. The latter, using spaced receivers, commonly employs FCA to obtain atmospheric parameters, such as wind velocity. The method devised by Briggs (1984) is widely applied, but in this paper we use the analysis carried out by Doviak et al. (1996) and Holloway et al. (1997b).

Both methods can be used to analyze data in anisotropic scattering conditions. However, simplifications are often made to the case of isotropic scattering, and furthermore, analyses of parameter estimate variance have been largely limited to simulations and analytical considerations of alongbaseline wind components (Tahara and Yamamoto, 1997; Kawano et al., 2002; Zhang et al., 2003).

In this paper we present the estimated variance of horizontal wind components for general anisotropic scattering, under which conditions the orientation of the baselines to the wind, as well as the orientation of the correlation ellipse, is arbitrary.

We assume a central transmitting antenna, and equi-distant but not co-linear receiver antennas. The magnitude of the normalized cross-correlation function $\rho_{i j}$ of received signals at spaced receiver antennas $i$ and $j$ are assumed to have a Gaussian form in the region where fitting is carried out.

The assumption of stationary atmospheric conditions was tested as follows. Gaussian curves were fitted to normalized auto-correlation functions and normalized cross-correlation functions of 26.2-s time series data. Gaussian curves were also fitted to normalized correlation functions of 262-s time series data, i.e. time series consisting of ten consecutive 26.2-s time series. The Gaussian curves for the ten 26.2-s data were compared to the curves for the 262-s data. For each auto-correlation and cross-correlation function, each of the ten 26.2-s fitted curves had to agree with the 262-s fitted curves to within the 262 -s fitted curve $95 \%$ confidence limits.

If all ten 26.2-s fitted curves agreed with the 262-s fitted curve to within the $95 \%$ confidence limits, then the 262-s fitted curves can be considered characteristic of the atmospheric refractive index fluctuations over the 262-s time interval, and also the data can be considered stationary. The outcome of the tests showed that the agreement was excellent (less than $0.5 \%$ difference), down to a correlation coefficients of 0.3 , which made the difference between the variance estimates using a Gaussian assumption and those using a direct measurement of correlation function magnitudes at lags of interest, negligible. In practice, if stationarity can be demonstrated, larger integration times (e.g. 262-s versus 26.2-s) can be used to collect data.

We describe the variance of horizontal wind components in terms of the un-normalized cross-correlation function $\mathrm{CCF}$ and auto-correlation function ACF magnitudes, following the example of Zhang et al. (2003). Characteristics of the atmospheric refractive index irregularities, such as backscattered power, correlation length and correlation time, and the radar parameters, such as baseline length, wavelength and transmitting and receiving antenna beam-width, determine the CCF magnitudes, and these magnitudes, in turn, determine the CCF magnitude variance and hence the estimated parameter (e.g. wind components, refractive irregularity correlation lengths) variance. 


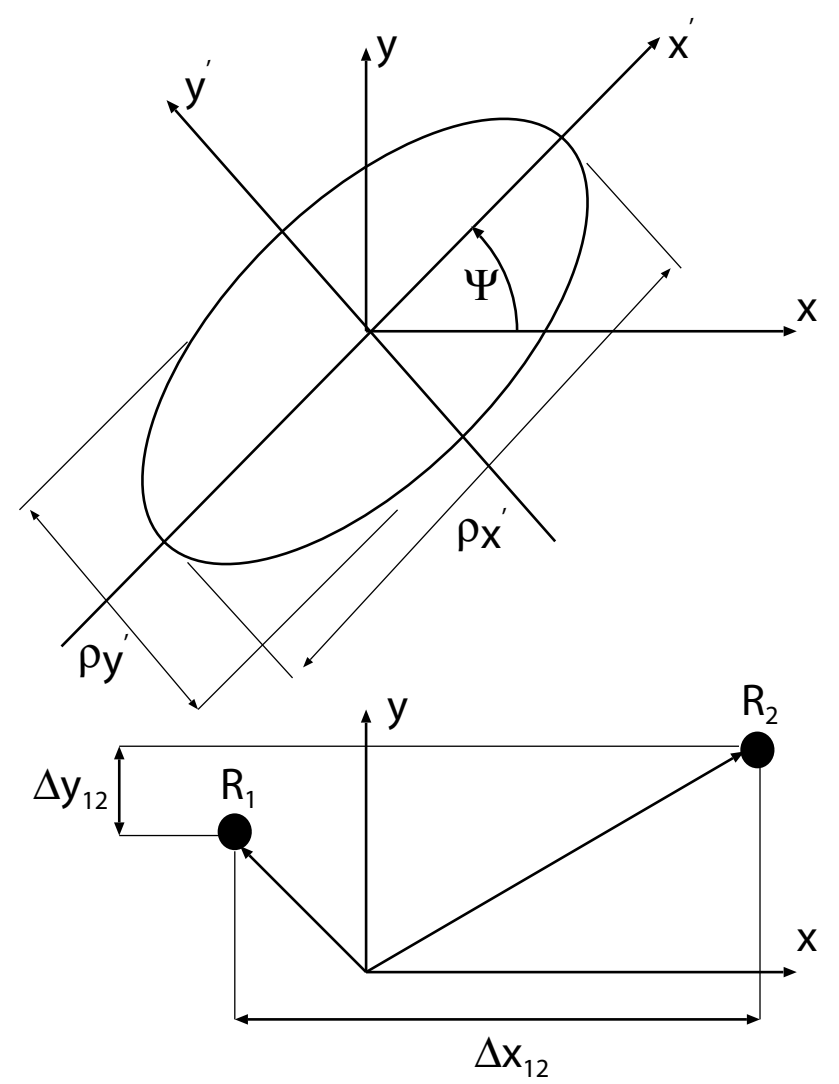

Fig. 1. The upper figure shows the two coordinate systems used. The axes labeled $x$ and $y$ define the ground coordinate system in which baselines are defined and horizontal wind components are measured. The axes labeled $x^{\prime}$ and $y^{\prime}$ define the coordinate system for the ground diffraction pattern and for the refractive index irregularity ellipse parameters $\rho_{x^{\prime}}$ and $\rho_{y^{\prime}}$. The two coordinate systems are related by the rotation angle $\Psi$. The lower figure shows the components $\Delta x_{12}$ and $\Delta y_{12}$ of an antenna baseline between two receiver antennas denoted by $R_{1}$ and $R_{2}$, in the ground coordinate system. For spaced antenna observations, three such baselines are defined between three non co-linear receiver antennas.

The analytical equations relate physical quantities $\sigma_{t}$ (fluctuation of vertical wind, often described as the assumed isotropic turbulence intensity), refractive index irregularity horizontal correlation lengths $\rho_{x^{\prime}}$ and $\rho_{y^{\prime}}$ with the orientation of the $x^{\prime}$-axis with respect to the $x$-axis given by $\Psi$, wind components $v_{x}$ and $v_{y}$ in the ground-based Cartesian coordinate system, and the radar antenna and baseline parameters. Figure 1 shows the relationship between the different axis systems, referring to the ground and the atmospheric scattering.

Results of our equations are compared to simulated results published by Kawano et al. (2002), and the differences are discussed. Finally we present data from the MU radar.

\section{Variance of wind components}

The normalized correlation ellipse, defining the horizontal statistics of atmospheric backscattering scales, is written as:

$\rho\left(\Delta x_{i j}, \Delta y_{i j}\right)=\rho\left(A \Delta x_{i j}^{2}+B \Delta y_{i j}^{2}+2 H \Delta x_{i j} \Delta y_{i j}\right)$

at time lag between receiver antennas $i$ and $j$ of $\tau_{i j}=0$, where the functional form of the normalized correlation function is left undetermined. $\Delta x_{i j}$ and $\Delta y_{i j}$ are orthogonal spatial lags (baseline components) measured from phase centers of receiver antenna numbers $i$ to $j$ in a Cartesian coordinate system, as illustrated in Fig. 1.

The governing equations for the wind components are depicted in Eq. (2) using normalized ACF and CCF magnitudes from two non co-linear baselines, to obtain an estimate for the two Cartesian wind components $v_{x}$ and $v_{y}$. A Gaussian functional form for the correlation functions is assumed:

$$
\begin{aligned}
& {\left[\begin{array}{c}
v_{x} \\
v_{y}
\end{array}\right]\left[\begin{array}{cc}
\left(A \Delta x_{i j} \tau_{i}^{(i j)}+H \Delta y_{i j} \tau_{i}^{(i j)}\right) & \left(B \Delta y_{i j} \tau_{i}^{(i j)}+H \Delta x_{i j} \tau_{i}^{(i j)}\right) \\
\left(A \Delta x_{j k} \tau_{i}^{(j k)}+H \Delta y_{j k} \tau_{i}^{(j k)}\right) & \left(B \Delta y_{j k} \tau_{i}^{(j k)}+H \Delta x_{j k} \tau_{i}^{(j k)}\right)
\end{array}\right]} \\
& =\left[\begin{array}{c}
\frac{1}{4}\left(A \Delta x_{i j}^{2}+B \Delta y_{i j}^{2}+2 H \Delta x_{i j} \Delta y_{i j}\right) \\
\frac{1}{4}\left(A \Delta x_{j k}^{2}+B \Delta y_{j k}^{2}+2 H \Delta x_{j k} \Delta y_{j k}\right)
\end{array}\right],
\end{aligned}
$$

where $\tau_{i}^{(i j)}$ is the time lag at the intercept of normalized ACF and CCF, as defined in Holloway et al. (1997a).

Assuming Gaussian forms for un-normalized correlation functions, we use the method described in Zhang et al. (2003) to derive the 10 variances and 45 covariances of unnormalized cross-correlation function magnitudes necessary to solve for wind components in a general SA configuration, with the restriction described in Sect. 1. The variances and covariances of the ACF and CCF magnitudes are considered for the infinite SNR case.

The main points of the derivation are as follows. First, the parameter of interest is written in terms of a combination of $\mathrm{ACF}$ and CCF magnitudes, instead of A, B, $2 \mathrm{H}$ and $\tau_{i}^{(i j)}$. Secondly, the variance of this parameter is derived by applying the standard theory of error propagation, taking all covariance terms into account. The covariances of the different ACF and CCF magnitudes at arbitrary time lags are derived using the method of Zhang et al. (2003). In such a manner, we obtain expressions for the variance of refractive index irregularity horizontal correlation lengths, ellipse orientation, and wind components.

\section{Effects of baseline length and orientation}

Figure 3 shows the analytical variance of wind speed estimated using three different pairs of baselines. As a reference, Fig. 2 shows the configuration used in the MU radar experiment described in Sect. 4. For the curves in Fig. 3, the baselines have been rotated so that $B_{23}$ is aligned with the $x$-axis, that is in the east-west direction, while the two other baselines sub-tend equal angles of $60^{\circ}$ on either side 
Table 1. Parameters for an experiment with the MU radar for comparison of experimental wind component variance with theoretically estimated variance. $\mathrm{D}$ is the transmitter antenna diameter, $\theta_{T x}$ and $\theta_{R x}$ are the transmitter and receiver antenna half-power beamwidth and field-of-view, respectively. Figure 2 shows the experiment antenna and baseline arrangement.

\begin{tabular}{lc}
\hline Time resolution & $26.2 \mathrm{~s}$ \\
Data points & 512 \\
Coherent Integrations & 128 \\
$\mathrm{D}$ & $103 \mathrm{~m}$ \\
$B_{12}=B_{13}=B_{23}$ & $52.0 \mathrm{~m}$ \\
$\theta_{12}$ & $137.48^{\circ}$ \\
$\theta_{13}$ & $-162.52^{\circ}$ \\
$\theta_{23}$ & $-102.52^{\circ}$ \\
$\theta_{T x}$ & $3.6^{\circ}$ \\
$\theta_{R x}$ & $10.1^{\circ}$ \\
\hline
\end{tabular}

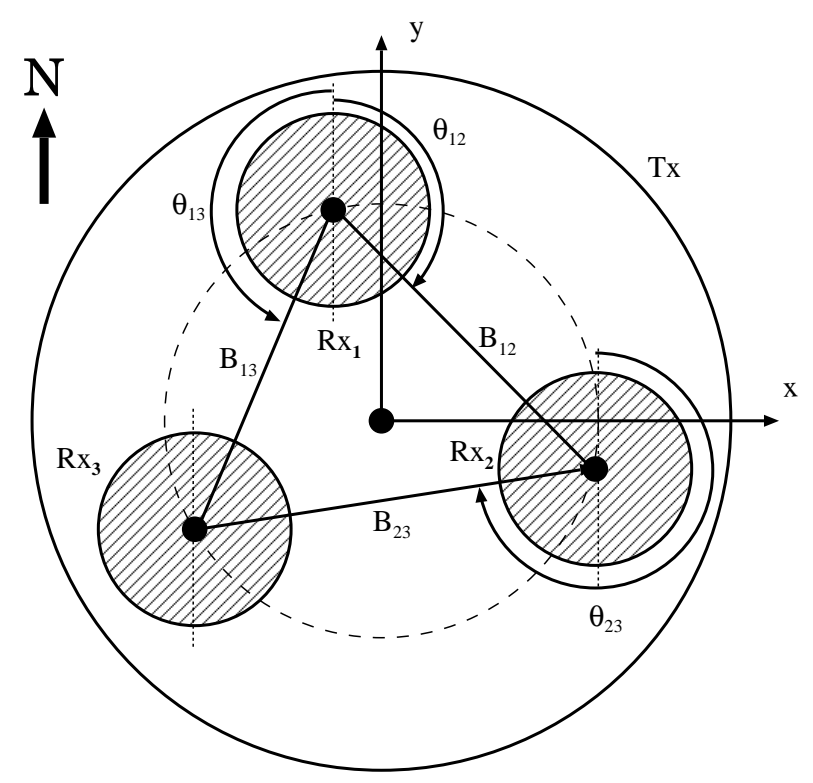

Fig. 2. MU radar antenna diagram showing receiver antenna phase centers located equi-distant from the transmitter antenna phase center.

of $B_{12}$. The wind direction was chosen to lie along the $x$ axis, that is along baseline $B_{23}$, with a zero $y$-component. For comparison with published numerical simulation results, data were collected in a series $175.1 \mathrm{~s}$ long. Other parameters are shown in Table 1. In Fig. 3, the different sets of curves for a single baseline pair are for different values of the correlation time $T_{0.5}$ (the correlation time of the backscattered signal power for an observer moving in the frame of reference of the mean horizontal atmospheric motion). This value is indirectly inversely related to the square root of the variance of vertical wind, $\sigma_{t}$, which is a measure of turbulence intensity. The wind component estimate variance depends on this parameter and on the geometry of the baselines.

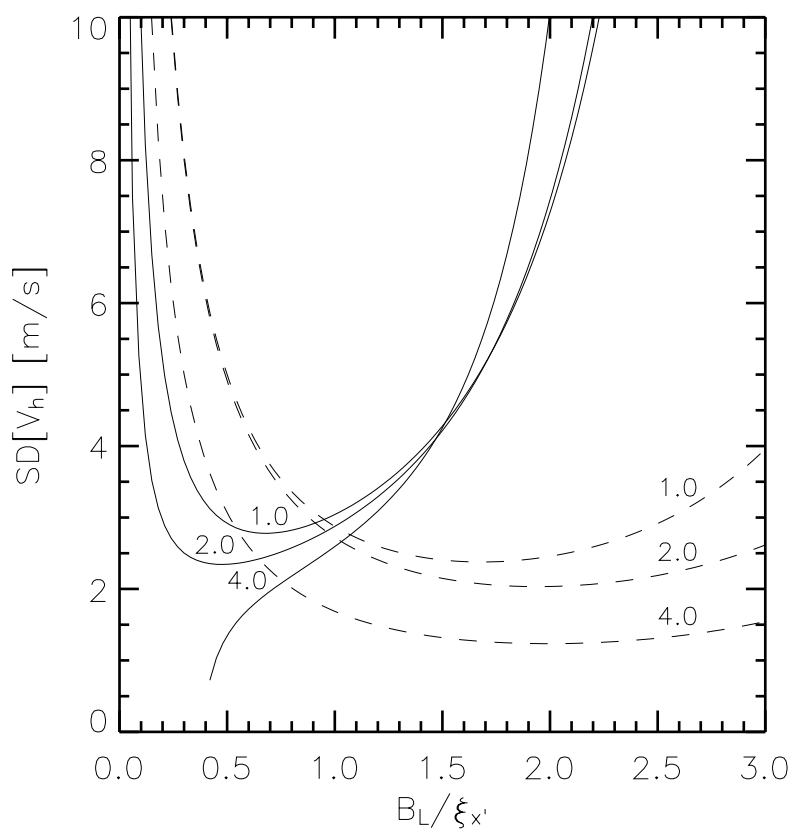

Fig. 3. Variance of wind $(30 \mathrm{~m} / \mathrm{s})$ as a function of isotropic diffraction pattern scale $\xi_{x^{\prime}}$ to baseline lengths $B_{i j}$ fixed at $B_{L}=52 \mathrm{~m}$. Correlation time $T_{0.5}$ varies from $1.0,2.0,4.0 \mathrm{~s}$. Results for baseline pair $\left(B_{13}, B_{12}\right)$ is shown in sold lines while results for baseline pairs $\left(B_{12}, B_{23}\right)$ and $\left(B_{13}, B_{23}\right)$ are shown in dashed lines. Observation time $T_{d}=175.1 \mathrm{~s}$.

The variance of wind estimates is related to CCF magnitudes. For a baseline perpendicular to the wind direction the CCF maximum will be lower compared to the CCF maximum along a baseline in the direction of the wind orientation. If Cartesian wind components $v_{x}$ and $v_{y}$ are found using Eq. (2), then for a wind oriented exactly along baseline $B_{23}$, the two sets of baselines with the greatest (and equal) alignment to this orientation, $\left(B_{12}, B_{23}\right)$ and $\left(B_{13}, B_{23}\right)$, show the lowest wind variance. However, baseline pair $\left(B_{12}, B_{13}\right)$ has a large difference in its orientation to the wind direction for each baseline, leading to a much larger estimated variance than the previous two sets. The dependence of variance on the ratio of baseline length to ground diffraction pattern scale, the Gaussian half-width $(1 / \sqrt{e})$ of the pattern, is due to the falling maximum CCF magnitude with increased spacing in the presence of $\sigma_{t} \neq 0$ (Holloway et al., 1997b). The pattern scale has been chosen here arbitrarily to be equal to the baseline length. Consequently, the horizontally-isotropic scatterer correlation length $(1 / \sqrt{e})$ is $9 \mathrm{~m}$.

As expected, small baseline lengths and low winds lead to poor estimation, the former because the CCFs are very similar to the ACFs and the intercept between them occurs in the flat portions of the curves near their peaks at small lag times, the latter because the CCF has already dropped to small values and the intercept between it and the ACF, once again, occurs in the flat portions of the curves at large lag times. This same issue holds for long baselines compared to ground diffraction pattern correlation lengths. 


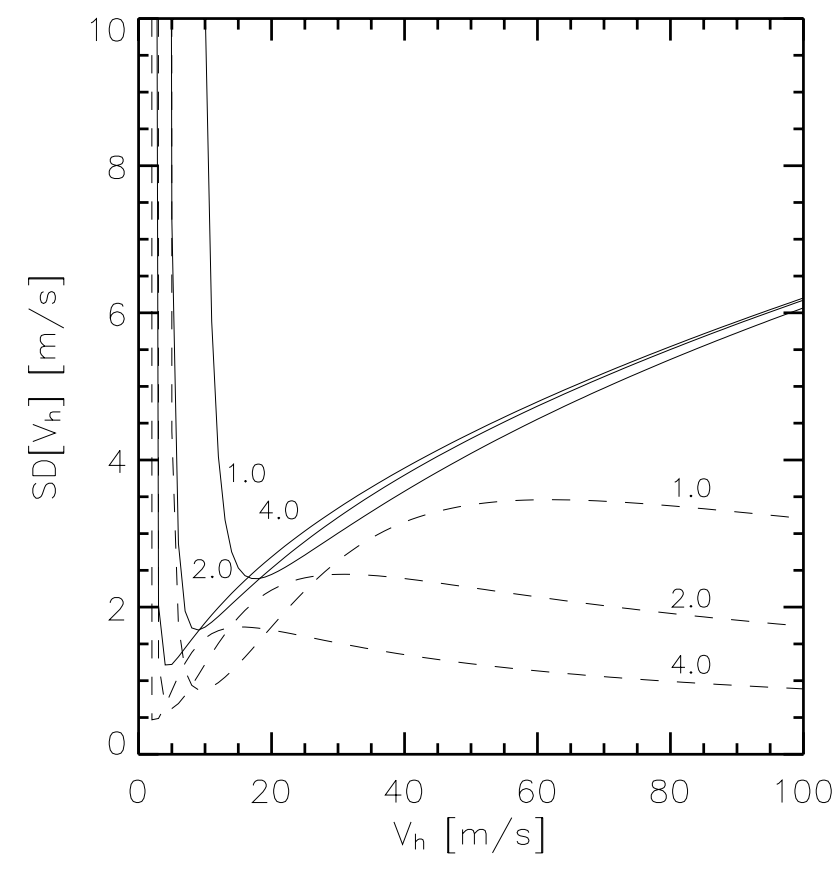

Fig. 4. The effect of wind speed on variance of the wind. $T_{0.5}$ is varied as before. Results for baseline pair $\left(B_{13}, B_{12}\right)$ is shown in sold lines while results for baseline pairs $\left(B_{12}, B_{23}\right)$ and $\left(B_{13}, B_{23}\right)$ are shown in dashed lines. $T_{d}=175.1 \mathrm{~s}, \xi_{x^{\prime}}=52 \mathrm{~m}, B_{i j}=52 \mathrm{~m}$.

The model correlation time $T_{0.5}$, related to the turbulence intensity, $\sigma_{t}$, of assumed isotropic turbulence, is varied from 1.0 -s to $4.0-\mathrm{s}$ to show how parameter estimation suffers with increased turbulence. Turbulence reduces correlation function peak values and width, thereby exacerbating the estimation problems at both extremes of long and short baselines, and narrowing the useful range of winds and baseline lengths over which estimation can be carried out to within a given variance.

In calculations for the variance of $\tau_{i}^{(i j)}$ for different baselines $B_{i j}$, the effect of the variance of $\tau_{c}$ on $\tau_{p}^{(i j)}$ was assumed negligible, following the results of Doviak et al. (2004). The model calculations become unwieldy as $\tau_{i}^{(i j)}$ becomes much larger than $\tau_{p}^{(i j)}+\tau_{c}$, where $\tau_{c}$ is the square root of the second moment of the Gaussian function. This situation occurs for low turbulence and is seen for the curve corresponding to $T_{0.5}=4.0-\mathrm{s}$ in Fig. 3.

Figure 4 shows how wind speed affects the wind estimate variance, due to the resulting shift in the CCF magnitude peak with wind speed. This effect is identical to that caused by varying the baseline length. The same conclusions about the effects of baseline orientation with respect to wind orientation, as described for Fig. 3, hold, namely that a minimum difference in alignment is conducive to smaller estimate variances. Time lag $\tau_{i}^{(i j)}$ at the intercept of normalized $\mathrm{ACF}$ and $\mathrm{CCF}$ is used in determining wind components in Eq. (2). The information included in $\tau_{i}^{(i j)}$ is the same as that of the CCF peak lag $\tau_{p}^{(i j)}$ in expressing the effects of base- line length and wind speed on the position and maximum of the CCF. Also, the decorrelation information contained in $\tau_{c}$ is included. $\tau_{i}^{(i j)}$ does not change with $\sigma_{t}$ (Holloway et al., 1997b), instead the influence on the estimate variances arises because as the ACF and CCF magnitudes (which determine $\tau_{i}^{(i j)}$ ) decrease with increasing turbulence, the variance of the position of the time lag increases. This is identical to the effect of $\tau_{x}^{(i j)}$, the lag time at which the auto-correlation function magnitude is equal to the zero-lag cross-correlation function magnitude, used in the Briggs method of FCA. $\tau_{x}^{(i j)}$ and $\tau_{i}^{(i j)}$ are related, as shown in Doviak et al. (2004).

The theoretical expressions for variance of estimated wind components in Figs. 3 and 4 can be compared with published simulation results with similar parameters in Kawano et al. (2002). The effects of $\tau_{i}^{(i j)}$ on the estimated variance cause an increase in variance of wind component estimation at low wind speeds, accentuated for higher turbulence (smaller $T_{0.5}$ ), which is not clearly visible in simulated along-baseline wind component results. Certainly our results show qualitative agreement with simulated results, also with the twodimensional simulation in Kawano (2000), where $T_{0.5}$ varies between 3.0-s and 9.0-s. In future work, the effect of SNR will be incorporated into the ACF and CCF magnitude variance and covariance estimates.

\section{Experimental comparison}

In order to test the analytical equations on real data, an experiment was carried out with the MU radar. Figure 2 shows the antenna and baseline arrangement used, while Table 1 gives the relevant radar parameters.

Time series of 26.2-s duration were collected for correlation analysis from $5.1 \mathrm{~km}-9.6 \mathrm{~km}$ at $150-\mathrm{m}$ resolution for correlation analysis. $\mathrm{CCF}$ and $\mathrm{ACF}$ were generated and checked for consistency against the 262-s average ACF and $\mathrm{CCF}$ at $95 \%$ confidence limits. Then, variances of CCF and ACF magnitudes of the 262-s time series of data were calculated and these combined to give wind component variance estimates. These calculated variances were compared to the variance of wind components estimated from the 26.2-s time series.

Figure 5 shows the results of the comparison of experiment with theory. The standard deviation (SD) of estimated zonal wind component $v_{x}$ was calculated from $N$ estimated values at 26.2-s resolution as follows:

$\mathrm{SD}\left[v_{x}\right]=\sqrt{\frac{\sum_{i=1}^{N / 2}\left(v_{x}[2 i]-v_{x}[2 i-1]\right)^{2}}{2(N / 2-1)}}$,

and similarly for the meridional component $v_{y}$. The wind had a peak magnitude of about $100 \mathrm{~m} / \mathrm{s}$, and a bearing close to $100^{\circ}$ from north. Accordingly, baseline pair $\left(B_{12}, B_{23}\right)$ was best positioned to measure the horizontal wind components, neatly bracketing the wind direction. The meridional 

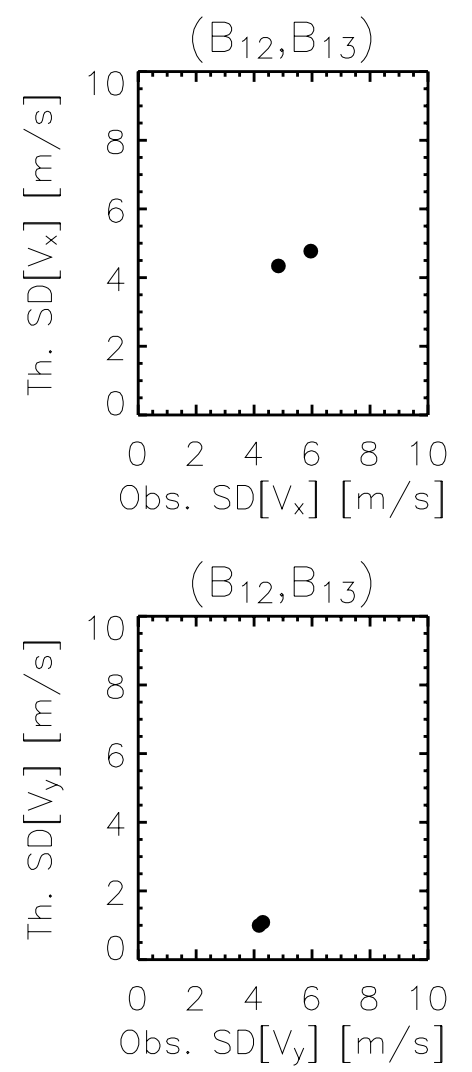
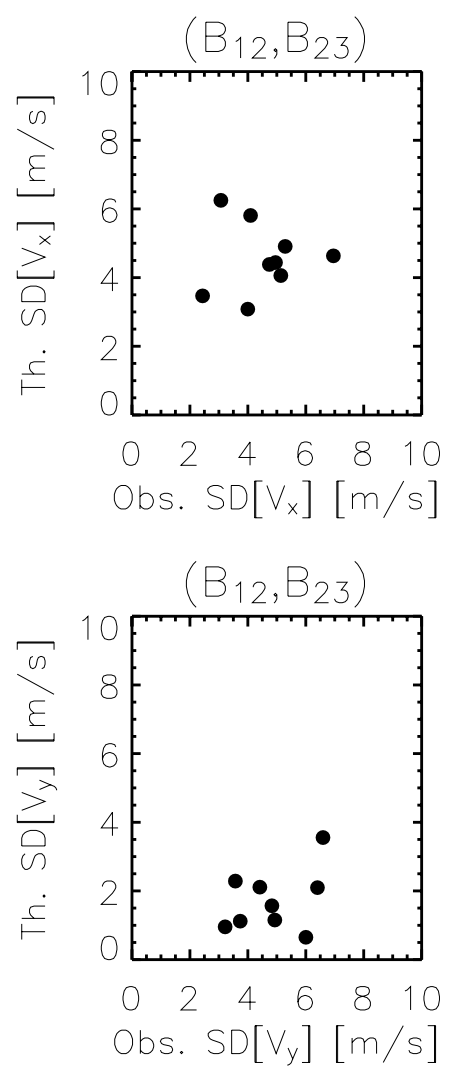
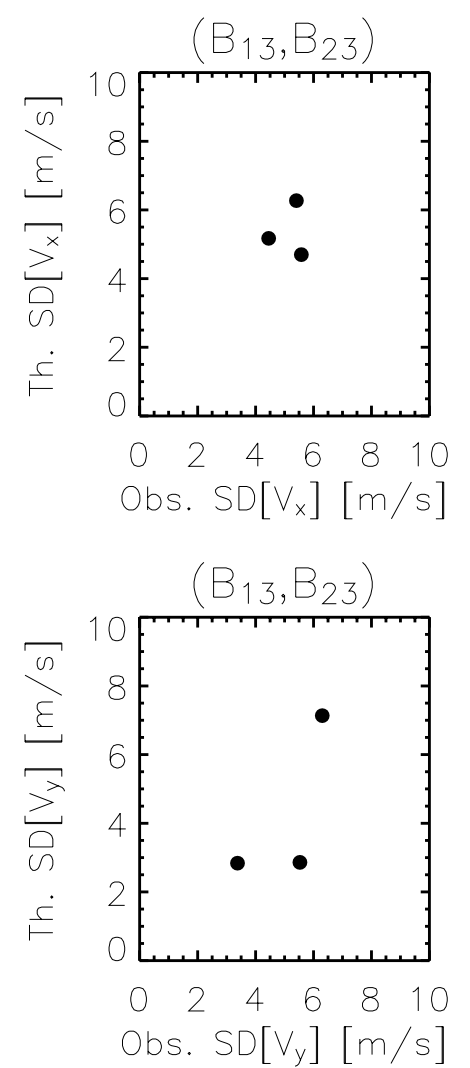

Fig. 5. A scatter plot showing the standard deviation (SD) of wind components from observation (Obs. SD) compared with theoretical calculations of the square root of the estimate variance (Th. SD), for different baseline pairs (see Fig. 2). Baseline pair $\left(B_{12}, B_{23}\right)$ brackets the average wind direction and shows best results for both $v_{x}$ and $v_{y}$.

component, $v_{y}$, is small and the number of data points limited, which may explain why the observed SD of this component was slightly greater than the theoretical SD value for this baseline pair. SNR above $20-\mathrm{dB}$ was chosen for the analysis, since low SNR is not yet accounted for in the theoretical equations.

\section{Conclusions}

In this paper we presented analytical solutions to the variance of wind estimates, applicable to two FCA-based SA techniques. The results hold for anisotropic conditions and are derived from the FCA analysis method of Doviak et al. (1996) and Holloway et al. (1997a), using cross-correlation function magnitude estimation, as per Zhang et al. (2003). The results are equally applicable to the Briggs method of FCA. In comparison with published simulations, the results showed good agreement, while an experiment with the MU radar confirmed that the theoretical wind variances qualitatively agreed with observed ones at high SNR $(>20-\mathrm{dB})$.

The variance of wind component estimates depends on the turbulence intensity (which is indirectly inversely proportional to correlation time $T_{0.5}$ ) through the mechanism of narrower $\mathrm{ACF}$ and $\mathrm{CCF}$, and reduced $\mathrm{CCF}$ magnitudes.
Furthermore, the baseline length affects the peak CCF magnitude lag, which thereby affects the wind component variance through varying peak CCF magnitude in the presence of turbulence, and changing $\tau_{i}^{(i j)}$ with correspondingly varying ACF and CCF magnitudes. The effect of finite SNR should be incorporated into $\mathrm{ACF}$ and $\mathrm{CCF}$ variance and covariance estimates in the future.

In experiments where multiple receivers are available, such as is the case with the upgraded MU radar from February 2004, the parameter estimations from many configurations can be compared. Analytical solutions for estimated parameter variance enable optimal configurations for given atmospheric conditions to be decided after the data has been collected, and checks can be carried out to determine whether the data is sufficiently stationary for theoretical variance estimates to be applicable.

Acknowledgements. The authors are indebted to R. J. Doviak and G. Zhang for their advice, support and encouragement. The MU radar belongs to and is operated by the Radio Science Center for Space and Atmosphere (RASC) of Kyoto University.

Topical Editor U.-P. Hoppe thanks two referees for their help in evaluating this paper. 


\section{References}

Briggs, B. H.: The analysis of spaced sensor records by correlation techniques, in Middle Atmosphere Program: Handbook for MAP, Vol.13, Ground-based Techniques, edited by R. Vincent, Univ. of Illinois, Urbana, 13, 166-186, 1984.

Doviak, R., Zhang, G. F., Cohn, S. A., and Brown, W. O. J.: Comparison of spaced-antenna wind estimators: Theoretical and simulated results, Radio Sci., 39, 2004.

Doviak, R. J., Lataitis, R. J., and Holloway, C. L.: Cross correlations and cross spectra for spaced antenna wind profilers 1 . Theoretical Analysis, Radio Sci., 31, 157-180, 1996.

Holloway, C. L., Doviak, R. J., and Cohn, S. A.: Cross correlations of fields scattered by horizontally anisotropic refractive index irregularities, Radio Sci., 32, 1911-1920, 1997a.

Holloway, C. L., Doviak, R. J., Cohn, S. A., Lataitis, R. J., and Van Baelen, J. S.: Cross correlations and cross spectra for spaced antenna wind profilers 2. Algorithms to estimate wind and turbulence, Radio Sci., 32, 967-982, 1997b.
Kawano, N.: Study of Spatial Domain Interferometry Technique with Atmospheric Radars, Ph. D. Thesis, Kyoto University, Japan, 2000.

Kawano, N., Tahara, Y., and Fukao, S.: Comparison of wind estimation errors for the spaced antenna technique: A case study for the MU radar, Radio Sci., 37, 2002.

Tahara, Y., Yamamoto, M., and Fukao, S.: Wind estimation errors of the spaced antenna technique studied with simulations and observations: A case study for the MU radar, Radio Sci., 32, 11931201, 1997.

Zhang, G., Doviak, R. J., Vivekanandan, J., Brown, W. O. J., and Cohn, S. A.: Cross-correlation ratio method to estimate crossbeam wind and comparison with a full correlation analysis, Radio Sci., 38, 8052-8063, 2003. 